\title{
Relation between cooperative molecular motors and active Brownian particles
}

\author{
Clément Touya, Tilo Schwalger, and Benjamin Lindner* \\ Max-Planck-Institut für Physik komplexer Systeme, Nöthnizer Straße 38, D-01187 Dresden, Germany
}

(Received 2 November 2010; published 16 May 2011)

\begin{abstract}
Active Brownian particles (ABPs), obeying a nonlinear Langevin equation with speed-dependent drift and noise amplitude, are well-known models used to describe self-propelled motion in biology. In this paper we study a model describing the stochastic dynamics of a group of coupled molecular motors (CMMs). Using two independent numerical methods, one based on the stationary velocity distribution of the motors and the other one on the local increments (also known as the Kramers-Moyal coefficients) of the velocity, we establish a connection between the CMM and the ABP models. The parameters extracted for the ABP via the two methods show good agreement for both symmetric and asymmetric cases and are independent of $N$, the number of motors, provided that $N$ is not too small. This indicates that one can indeed describe the CMM problem with a simpler ABP model. However, the power spectrum of velocity fluctuations in the CMM model reveals a peak at a finite frequency, a peak which is absent in the velocity spectrum of the ABP model. This implies richer dynamic features of the CMM model which cannot be captured by an ABP model.
\end{abstract}

DOI: 10.1103/PhysRevE.83.051913 PACS number(s): 87.10.Mn, 05.10.Gg, 05.40.Jc, 87.16.Nn

\section{INTRODUCTION}

Active (self-propelled) motion is one of the obvious signatures of many living systems and can be observed on different levels, ranging from the motion of motor proteins within cells to the motility of cells and bacteria $[1,2]$. Although active motion is in most cases inherently stochastic, its statistics differ from those of equilibrium "passive" Brownian particles, because by one or the other sort of energy consumption the active system is brought out of thermodynamic equilibrium.

The detailed (physical or biochemical) mechanism giving rise to such an active pumping mechanism can be quite complicated. Therefore, theoreticians mainly interested in collective phenomena in systems of interacting particles (see, e.g., [3,4] and references therein) have used simple phenomenological models such as, for instance, the popular Vicsek model [5], in which only the velocity's angle but not its absolute value (the speed) is subject to noise and interactions. As an alternative, more detailed phenomenological model, the active Brownian particle has been used to describe biological motility in different nonequilibrium situations [6-9]. In a one-dimensional version, this model is given by a Langevin equation for the particle's velocity that contains a velocitydependent drift term and a noise with a velocity-dependent amplitude:

$$
\dot{x}=v, \quad \dot{v}=f(v)+g(v) \xi(t) .
$$

Here, $\xi(t)$ is a Gaussian white noise of intensity $Q$ [i.e., $\left.\left\langle\xi(t) \xi\left(t^{\prime}\right)\right\rangle=2 Q \delta\left(t-t^{\prime}\right)\right]$, and the stochastic differential equation is here and in the following always interpreted in the Ito sense.

A nonlinear Langevin equation such as Eq. (1) can also describe an equilibrium situation if a generalized Einstein relation is satisfied [10,11]. For an active Brownian particle (ABP), as considered in the following, the nonlinear dissipation term $f(v)$ is negative for a range of velocities, which precludes thermodynamic equilibrium. Several authors have measured

*Corresponding author: benji@pks.mpg.de the functions $f(v)$ and $g(v)$ (also in multidimensional setups) from experimental data of biological systems [6,12-14].

Besides the phenomenological description of experimental data, ABP models have been shown to reproduce qualitatively the statistics of more complicated multivariable stochastic models $[14,15]$. One such model proposed by Jülicher and Prost [16] describes the stochastic dynamics of a group of rigidly coupled molecular motors (CMMs) [15,17].

A novel collective effect seen in systems of many CMMs is a bidirectionality of motion, which was also found experimentally in motility essays [18]. Features of the CMM's diffusional behavior could be predicted using the simpler ABP model [17]. This motivates to study the relation between the CMM model and the ABP model in more detail. Specifically, we would like to know whether and if so which functions $f(v)$ and $g(v)$ in Eq. (1) can describe the stochastic dynamics of the CMM model.

A rigorous mathematical connection between the CMM and ABP models, i.e., an (approximate) mapping from one model to the other, is still lacking. In this paper we provide numerical versions of this mapping. We use two independent numerical approaches: one which is based on the stationary velocity distribution of the motor system and one which is based on the Kramers-Moyal coefficients of the velocity (the two approaches were used by Yates et al. [14], who studied experimental data from locusts). The fact that both methods yield comparable results for the functions $f(v)$ and $g(v)$ underpins that the ABP model is a reasonable approximation of a system of many coupled motors. However, we also discuss results that show the limitations of the ABP model as an approximation and demonstrate that the CMM model is more complex than the former model.

Our paper is organized as follows. In the next section, we recall the details of the CMM model, illustrate its dynamics for a finite number of motors, and remind the reader of some analytical results for the thermodynamic limit $(N \rightarrow \infty)$. In Sec. III we explain the two methods by which we extract numerically drift and noise amplitude from simulations of the CMM model. In Sec. IV we present these functions for different cases and study the accuracy of the ABP 


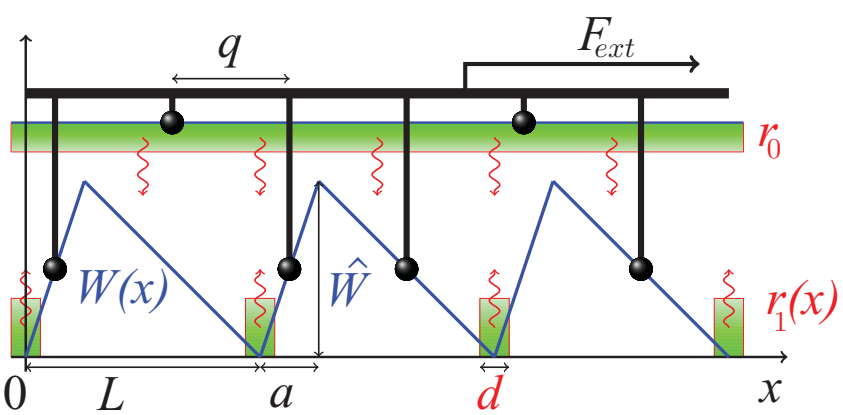

FIG. 1. (Color online) Model of the coupled molecular motors as introduced in $[15,16]$.

approximation by different means. Here we also compare the drift function to the force-velocity relation previously obtained in the thermodynamic limit. By looking at the power spectra, we show the limitations of such a mapping and we illustrate it with one special situation. We summarize our results and discuss open questions in Sec. V.

\section{THE MODEL OF COUPLED MOLECULAR MOTORS (CMM) AND ITS THERMODYNAMIC LIMIT}

The model is illustrated in Fig. 1. It consists of $N$ particles (motors), which are coupled rigidly to a backbone (with fixed nearest-neighbor distance $q$ ) and can switch between two biochemical states associated with two different free energy landscapes, one of which is flat (no potential forces). The potential in state 1 is called $W(x)$ and is given by the piecewise linear ratchet potential shown in Fig. 1 by the blue line. The potential has period $L$ and possesses a maximum of height $\hat{W}$ at $x=a$; the symmetric case corresponds to $a=L / 2$. The on-rate $r_{0}$ is independent of the position of the motor, whereas the off-rate $r_{1}(x)$ is space-dependent and attains a finite constant value $\hat{r}_{1}$ only in a region of width $d$ centered around the potential's minimum (at $x=0) ; r_{1}(x)$ is shown in Fig. 1 by the lower red line.

The overdamped motion of the backbone results from the force balance of frictional forces, thermal forces, and potential forces on the motors:

$$
\begin{gathered}
\lambda \dot{x}=F_{\text {ext }}-\frac{1}{N} \sum_{j=1}^{N} \sigma_{j}(t) W^{\prime}(x+j \cdot q)+\sqrt{\frac{k_{B} T \lambda}{N}} \eta(t) \\
\sigma_{j}=0 \underset{r_{1}(x+j \cdot q)}{\stackrel{r_{0}}{\rightleftharpoons}} \sigma_{j}=1
\end{gathered}
$$

where $\lambda$ is a an effective friction coefficient per motor and $F_{\text {ext }}$ an external force applied to the backbone. The state of a given motor $j$ at time $t$ is given by the variable $\sigma_{j}(t)$ which takes the values 0 or 1 ; switchings of the motor state take place according to the above discussed rates $r_{\{0,1\}}$. If not stated otherwise, the parameters used in the following are (in nondimensional units) $L=1, a=L / 2$ (symmetric case), $d=0.2 L, \hat{W}=1$, and $\lambda=0.01$. Moreover, the rates are taken to be $r_{0}=40$ and $\hat{r}_{1}=500$. For simplicity, in the following we neglect the thermal fluctuations.

The model can be simulated as follows. In every time step $\Delta t^{\mathrm{sim}}$, we estimate the probability of each motor to perform a transition depending on its current state and possibly on its position along the backbone. A motor that is, for instance, in state 1 and located within the narrow region around the minimum has the probability $p=r_{1} \Delta t^{\text {sim }}$ to switch to state 0 . In our simulations, a random number $\eta_{i}$ between 0 and 1 is drawn. If this is smaller than $p$ the transition is performed; otherwise, the motor remains in its state. After this has been done for every motor, Eq. (2) is integrated by one time step (for all data shown in this paper, we have used $\Delta t^{\mathrm{sim}}=10^{-4}$ ). In this way, long trajectories of position and velocity $v=\dot{x}$ of the motor assembly can be simulated and used to estimate various quantities of interest.

Because of the switching, already a single motor can perform a directed motion even in the absence of an external force. For this to occur, the underlying spatial potential should be asymmetric (ratchet potential with $a \neq L / 2$ ). Many motors will also show a directed motion. The dynamics of many motors, however, displays a new collective effect: The assembly of motors runs in one direction even for a symmetric potential $(a=L / 2)$ but switches the sign of the velocity from time to time in a stochastic manner. This is called a bidirectional motion; it can be also observed in experiment, and it can also occur if the system is asymmetric but biased against its preferred direction by an external force [15].

With increasing number of motors, the typical time for a velocity reversal increases exponentially. In the thermodynamic limit, no reversals occur at all. The system suffers a spontaneous symmetry breaking, and for a range of external forces, there are two stable values of the velocity which are approached depending on the initial conditions of the system. Another manifestation of this fact is a nonlinear (inverted-N-shaped) force-velocity relation.

Mathematically, the system simplifies considerably in the thermodynamic limit and becomes tractable, such that the force-velocity relation can be calculated analytically. Here we want to briefly recall the basic equations and results because we will also use them in the remainder of the paper.

In the limit of $N \rightarrow \infty$, the total density of motors lumped into one period becomes uniform, i.e.,

$P(x)=P_{0}(x)+P_{1}(x)=\lim _{\substack{N \rightarrow \infty \\ \Delta x \rightarrow 0}} \frac{n_{1}(x)}{N \Delta x}+\frac{n_{0}(x)}{N \Delta x}=\frac{1}{L}$,

where $n_{0,1}(x)$ are the number of motors in $[x-\Delta x / 2, x+$ $\Delta x / 2]$ which are in state 0 or 1 , respectively. The two densities obey the equations [16]

$$
\begin{gathered}
\partial_{t} P_{1}+v \partial_{x} P_{1}=-r_{1}(x) P_{1}(x)+r_{0} P_{0}(x) \\
\partial_{t} P_{0}+v \partial_{x} P_{0}=r_{1}(x) P_{1}(x)-r_{0} P_{0}(x),
\end{gathered}
$$

which can be recast into only one equation for $P_{1}(x)$ by means of the normalization condition Eq. (3). The density should be periodic in $x$, i.e., $P(0)=P(L)$. The velocity $v$ is determined by the distribution of motors in the state 1 by the following formula:

$$
v(t)=\frac{1}{\lambda}\left[F_{\mathrm{ext}}-\int_{0}^{L} d x W^{\prime}(x) P_{1}(x, t)\right] .
$$

In the steady state, Eq. (4) combined with the normalization condition Eq. (3) leads to

$$
v \partial_{x} P_{1}^{s t}(x)=-\left[r_{1}(x)+r_{0}\right] P_{1}^{s t}(x)+r_{0} / L .
$$


Suppose we know the velocity of the backbone; one can then calculate the general solution [19]

$$
P_{1}^{s t}(x)=r_{0} \frac{\int_{x}^{x+L} d y \exp \left(\int_{x}^{y} d z\left[r_{1}(z)+r_{0}\right] / v\right)}{L v\left\{\exp \left(\int_{0}^{L} d z\left[r_{1}(z)+r_{0}\right] / v\right)-1\right\}} .
$$

The function $v$ is determined self-consistently from Eq. (5) with $P_{1}(x, t)=P_{1}^{s t}(x)$. It can be formally inverted with respect to $F_{\text {ext }}$

$$
F_{\text {ext }}=\hat{f}(v)=\lambda v+\int_{0}^{L} d x W^{\prime}(x) P_{1}^{s t}(x)
$$

This equation states which external force would be required to see a certain (prescribed) steady-state velocity; it yields in general a nonmonotonic force-velocity relation $\hat{f}(v)$ that is shaped like an inverted $\mathrm{N}$.

\section{METHODS FOR EXTRACTION OF DRIFT TERM AND NOISE AMPLITUDE}

Let us assume in this section that a given system out of equilibrium can indeed be described by an ABP process, and thus by the nonlinear Langevin Eq. (1). Given a large amount of data, which could come from simulations of the microscopic model or from experiments, one can extract the functions $f$ and $g$ by two independent methods. They are based, respectively, on the short and long time behavior of the system. Agreement of both methods would indicate that the ABP model is a good model for the given data. Further evidence can be found by a comparison of the stationary velocity probability density between the data and the ABP model, and by measuring dynamic quantities, such as the effective diffusion coefficient, or correlations and power spectra. Here we apply this program to the CMM model.

Our method here is similar to the one used by Yates et al. [14], who extracted the functions $f(v)$ and $g(v)$ by measuring the Kramers-Moyal coefficients and compared the resulting stationary probability density (see below) to the velocity's histogram. In contrast to this, we extract $f(v)$ and $g(v)$ from both kinds of statistics independently.

\section{A. Extracting $f(v)$ and $g(v)$ from the stationary velocity probability density (distribution method)}

The first method (in the following referred to as the distribution method) is based on the stationary distributions $P_{0}(v)$ of the process $v$. If we interpret Eq. (1) in the sense of Ito, the distribution $P(v, t)$ obeys the following Fokker-Planck equation [20]:

$$
\partial_{t} P(v, t)=\partial_{v}\left\{\left[-f(v)+Q \partial_{v} g^{2}(v)\right] P(v, t)\right\},
$$

with the asymptotic solution at long times

$$
P_{0}(v)=C_{Q} \frac{\exp \left[-Q^{-1} U(v)\right]}{g^{2}(v)},
$$

where $C_{Q}$ is a normalization constant, and the function $U(v)$ is an effective potential. They are given by

$$
C_{Q}=\left(\int_{-\infty}^{\infty} d v \frac{\exp \left[-Q^{-1} U(v)\right]}{g^{2}(v)}\right)^{-1}
$$

and

$$
U(v)=-\int_{0}^{v} d \tilde{v} \frac{f(\tilde{v})}{g^{2}(\tilde{v})}+U_{0},
$$

respectively. In this framework, the effective potential is defined up to a constant $U_{0}$. We may fix this constant by requiring the potential to be zero at a specific value of the velocity.

Can we extract the functions $f(v)$ and $g(v)$ from one or several velocity histograms? First of all, it is possible to obtain the effective potential from two histograms measured at different noise levels $Q_{1}$ and $Q_{2}$ as follows. Taking the logarithms of the ratio of the two histograms, we obtain according to Eq. (10)

$$
\ln \left(\frac{P_{0, Q_{1}}(v)}{P_{0, Q_{2}}(v)}\right)=\ln \left(\frac{C_{Q_{1}}}{C_{Q_{2}}}\right)-\left(Q_{1}^{-1}-Q_{2}^{-1}\right) U(v),
$$

where the function $g^{2}(v)$ cancels. From this, one can numerically extract the function $U(v)$ up to a constant

$$
U(v)=-\frac{\ln \left(P_{0, Q_{1}}(v)\right)-\ln \left(P_{0, Q_{2}}(v)\right)}{Q_{1}^{-1}-Q_{2}^{-1}}+\tilde{U}_{0} .
$$

How do we change the noise intensity in the CMM model? From general considerations [15], it is plausible that the noise intensity is set by the inverse number of motors,

$$
Q=1 / N,
$$

i.e., $Q_{1}^{-1}-Q_{2}^{-1}=N_{1}-N_{2}$. Note that in our ABP approximation, this is the only place where the number of motors enters. The noise amplitude is split into two factors: the purely velocity-dependent function $g(v)$ and the $N$-dependent factor $Q$. We emphasize that in our ABP approximation both $f(v)$ and $g(v)$ are assumed to be independent of $N$.

Because $U(v)$ depends only on $f(v)$ and $g(v)$ [cf. Eq. (12)], we should then get the same potentials for any two values of $N_{1}$ and $N_{2}$ from Eq. (14). This is indeed what we find and

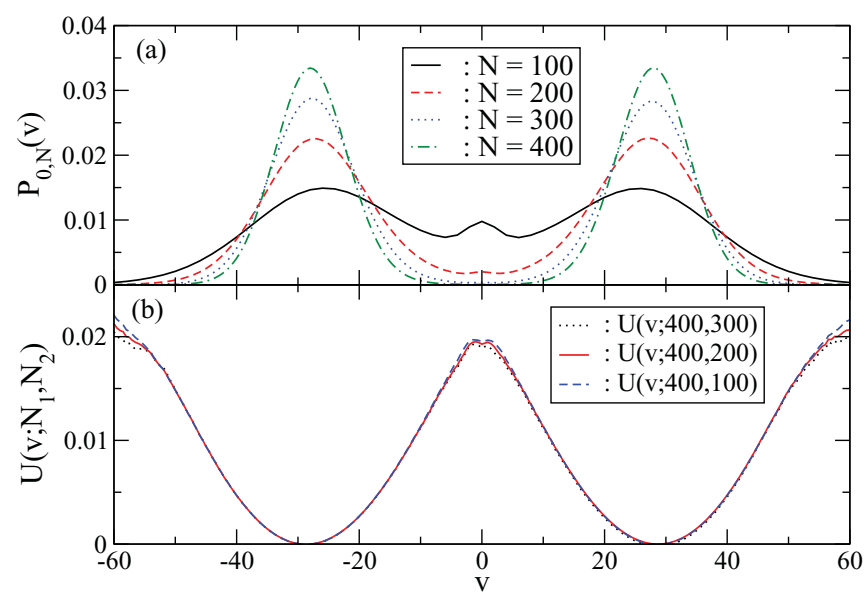

FIG. 2. (Color online) Results for the two-state model for an assembly of $N$ motors in the symmetric case $(a=0.5 L)$, without external forces $\left(F_{\text {ext }}=0\right)$. (a) Normalized stationary distribution $P_{0, N}$ for $N=400,300,200$, and 100. (b) Effective potential $U\left(v, N_{1}, N_{2}\right)$ for various combinations of $N_{1}$ and $N_{2}$. They all collapse perfectly into one curve as expected. (We have shifted the potential so that the minima correspond to 0 .) 
what is illustrated in Fig. 2. Histograms for four different values of $N$ [see Fig. 2(a)] yield potentials which, if properly shifted, agree rather well, as shown in Fig. 2(b). The effective potential found in this way is bistable and symmetric, with the two minima corresponding to the most probable velocities of the bimodal distributions in Fig. 2(a). Further evidence and also the limitations of the assumption about the independence of $f(v)$ and $g(v)$ of $N$ are inspected below in Sec. IV A.

Rewriting Eq. (10), we can express $g(v)$ in terms of the effective potential, the probability density, and the normalization constant,

$$
g(v)=\sqrt{\frac{C_{Q} \exp \left[-Q^{-1} U(v)\right]}{P_{0, Q}(v)}} .
$$

This formula does not suffice to obtain $g(v)$, because we do not know the normalization constant. We have to use an extra piece of information from the CMM system. It turns out that the effective diffusion coefficient $D_{\text {eff }}$ of the CMM model defined by

$$
D_{\mathrm{eff}}=\lim _{t \rightarrow \infty} \frac{1}{2 t}\left(\left\langle x^{2}(t)\right\rangle-\langle x(t)\rangle^{2}\right)
$$

is sufficient to determine the missing factor. For an ABP we know an explicit expression for this characteristic in terms of $f(v)$ and $g(v)[17]:$

$$
D_{\text {eff }}=\frac{C_{Q}}{Q} \int_{-\infty}^{\infty} d x e^{U(x) / Q}\left[\int_{-\infty}^{x} d y(y-\langle v\rangle) \frac{e^{-U(y) / Q}}{g^{2}(y)}\right]^{2},
$$

where $\langle v\rangle=\int d v P_{0}(v) v$ denotes the average velocity. Using the definition of $P_{0, Q}$, we can now rewrite Eq. (18) and find the following expression for $C_{Q}$ in terms of the diffusion coefficient:

$$
C_{Q}=\frac{Q^{-1}}{D_{\mathrm{eff}}} \int_{-\infty}^{\infty} d x e^{U(x) / Q}\left[\int_{-\infty}^{x} d y(y-\langle v\rangle) P_{0, Q}(y)\right]^{2} .
$$

Using $D_{\text {eff }}, U(v)$, and $P_{0, Q}(v)$, we can thus determine $C_{Q}$ and via Eq. (16) the function $g(v)$. Furthermore, differentiating Eq. (12), we obtain a simple formula for $f(v)$,

$$
f(v)=-g^{2}(v) U^{\prime}(v),
$$

where the prime denotes differentiation with respect to $v$.

In conclusion, what we need in order to find the functions $f(v)$ and $g(v)$ for the CMM model are at least two velocity histograms taken at different numbers $N_{1}$ and $N_{2}$ of motors and a numerical estimate of the spatial diffusion coefficient at one number $N_{D}$; in the following, we use $N_{D}=N_{1}$. More simulation data (e.g., for different numbers of motors) can then be used to verify the consistency of the ABP approximation.

\section{B. Extracting $f(v)$ and $g(v)$ via the Kramers-Moyal coefficients of the velocity (increment method)}

If the ABP model is a faithful description of the velocity's time series, mean and variance of the increments should be related to the two functions $f(v)$ and $g^{2}(v)$, respectively. Put differently, by estimating the so-called Kramers-Moyal coefficients [20], we can obtain an independent estimate of the desired functions.

If we discretize Eq. (1) to the first order in $\Delta t$ (not to be confused with the simulation time step $\Delta t^{\text {sim }}$ of the CMM model), we have

$$
v(t+\Delta t)-v(t) \approx f(v(t)) \Delta t+g(v(t)) \sqrt{2 Q \Delta t} \eta_{i},
$$

where $\eta_{i}$ are uncorrelated Gaussian numbers of zero mean and unit variance $\left\langle\eta_{i} \eta_{j}\right\rangle=\delta_{i, j}$. As is well known [20], mean and variance of these increments define the desired functions by

$$
f(v)=\left\langle\frac{v(t+\Delta t)-v(t)}{\Delta t}\right\rangle_{v(t)=v}
$$

and

$$
g^{2}(v)=\frac{\Delta t}{2 Q}\left\langle\left(\frac{v(t+\Delta t)-v(t)}{\Delta t}-f[v(t)]\right)^{2}\right\rangle_{v(t)=v} .
$$

The two functions are estimates of the first two of the KramersMoyal coefficients defined by

$$
D^{(n)}(v)=\frac{1}{n !} \lim _{\Delta t \rightarrow 0} \frac{1}{\Delta t}\left\langle[v(t+\Delta t)-v]^{n}\right\rangle_{v(t)=v},
$$

or, more specifically,

$$
f(v) \approx D^{(1)}(v), \quad g^{2}(v) \approx N D^{(2)}(v) .
$$

For a diffusion process like that of the ABP model, coefficients with $n>2$ should vanish.

An important question is which sampling time step $\Delta t$ we should choose in Eqs. (22) and (23) in order to obtain reasonable estimates of $f(v)$ and $g(v)$. In close analogy to the discussion of the so-called chemical Langevin equation by Gillespie [21], one can argue that the increments of the velocity will only be Gaussian distributed if we see enough switching events within the time step $\Delta t$, which would suggest to use a large time step. On the other hand, in order to obtain a meaningful estimate of the instantaneous drift and diffusion coefficients, the velocity should change only little within $\Delta t$. These considerations lead to the following rough estimate for the recommendable time step:

$$
\frac{1}{N r_{0}} \ll \Delta t \ll \frac{1}{r_{0} / 2+\hat{W} / \lambda L^{2}},
$$

where we have used an estimate for the number of transitions $N r_{0} \Delta t$ (using the lower rate $r_{0}<r_{1}$ but assuming all particles in the unbound state) and have estimated an upper bound for the maximal change in velocity during $\Delta t$. The inequality simply illustrates that for a sufficiently large number of motors, we should be able to find a $\Delta t$ satisfying the above condition. In our numerical simulations, we have used $\Delta t=10^{-3}$, which satisfies the inequality (26) for our parameters.

\section{NUMERICAL RESULTS FOR $f(v)$ AND $g(v)$ OF THE CMM MODEL}

In this section we compare the results for the effective ABP model from both methods for three cases: (i) a symmetric potential $W(x)$, (ii) a symmetric potential with an additional bias force, and (iii) an asymmetric potential without bias force. We also discuss whether the drift $f(v)$ coincides with the forcevelocity relation measured previously [15], and we inspect 
numerically how a bias force in the CMM model translates into a bias force in the effective ABP dynamics. Finally, we study the relaxation of the velocity from extremal values.

\section{A. Spatially symmetric case $\left(a=L / 2, F_{\mathrm{ext}}=0\right)$}

We start here with the simplest case, where the ratchet potential is symmetric $(a=0.5 L)$ and no external force is applied $\left(F_{\text {ext }}=0\right)$. For large $N$, the velocity distributions shown in Fig. 2(a) are symmetric and clearly bimodal and peaked around two values $v_{ \pm}^{\star} \approx \pm 28$, reflecting the stochastic switching between positive and negative velocities, i.e., the bidirectional motion of the backbone. For small $N$ (typically around $N \sim 100$ ), a small local maximum at zero velocity is observed.

From two of the densities and the diffusion coefficient at one motor number, we can obtain the effective potential and the functions $f(v)$ and $g(v)$. Independently, we can measure $f(v)$ and $g(v)$ via the Kramers-Moyal coefficients as explained in the previous section. The results of both methods agree rather well for the symmetric case, as illustrated in Fig. 3.

The potential obtained by the distribution method is clearly bistable. The function $f(v)$ has the expected shape of an inverted $N$ (similar to a cubic function). Most remarkably, the noise intensity $g(v)$ also depends on the speed: it is smallest at zero velocity and increases toward larger speed. For $g(v)$, we find small differences between the methods, whereas the agreement for $f(v)$ is excellent. We would like to point out that $g(v)$ determined via the increment method depends to a certain extent on the time step of the procedure [see discussion around Eq. (26)].

If our ABP approximation is meaningful, all the dependence of the ABP dynamics on the motor number $N$ should be in the noise prefactor $Q$ according to Eq. (15). In other words, $f(v)$ and $g(v)$ as determined from the CMM model should be the same irrespective of the number of motors used in the simulation. This assertion is inspected in Fig. 4 for the speed dependence of drift and noise amplitude determined

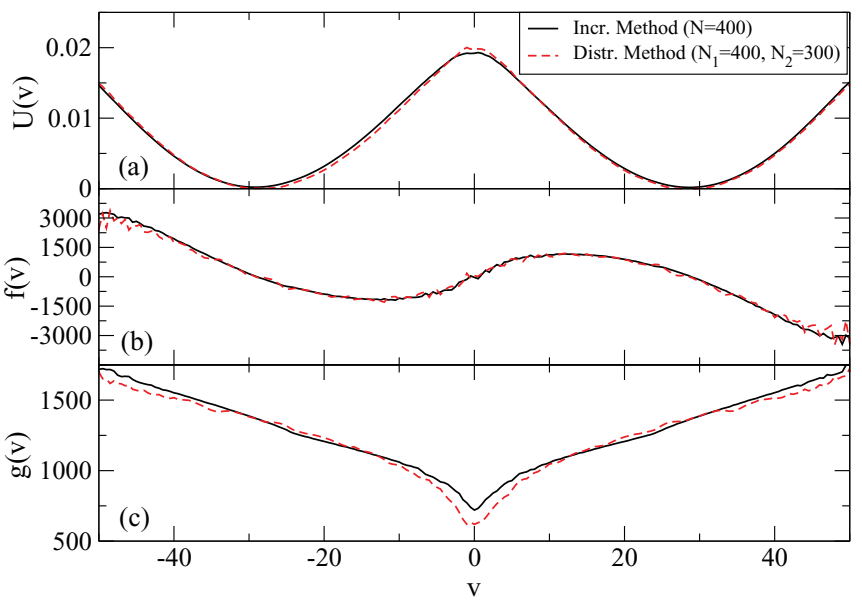

FIG. 3. (Color online) Effective potential (a), drift (b), and noise amplitude (c) of the CMM model in the symmetric case without external forces. Functions were determined by the distribution method (with $N_{1}=400, N_{2}=300$, dashed line) and increment method (with $N=400$, solid line).

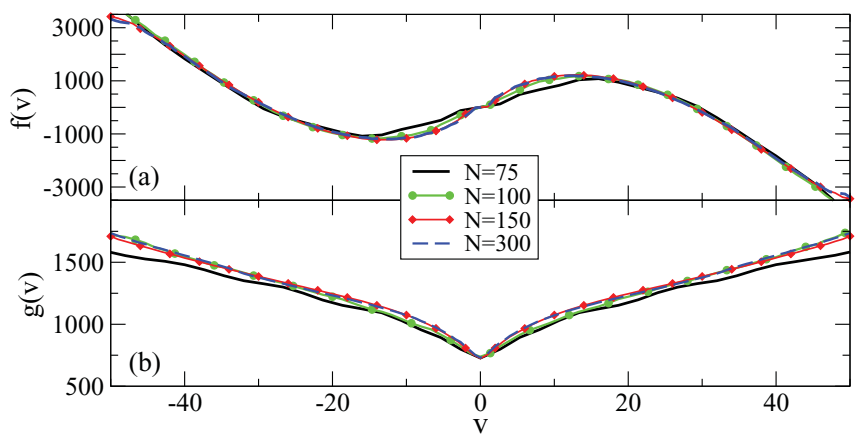

FIG. 4. (Color online) Drift (a) and noise amplitude (b) of the CMM model (increment method) for different numbers of motors as indicated.

via the increment method. For $N>100$, we do observe a good agreement of the curves, consistent with the ABP approximation in which the $N$ dependence is solely carried by the prefactor $Q$. Drift terms and noise amplitudes for $N=150$ and $N=300$ virtually agree with each other; they also agree with $N=400$ (not shown). However, data for $N=100$ show slight and for $N=75$ significant deviations in both $f(v)$ and $g(v)$ compared to what we obtained for larger $N$. First of all, the overall noise is slightly reduced [e.g., $g_{N=75}(v)<g_{N=150}(v)$ for all $v$ ]. The effect on the drift, however, is more drastic in that the nonlinearity [in particular, between the minimum and the maximum of $f(v)$ ] is flattened out. We conclude that the mapping from CMM to the ABP model is constrained to motor numbers larger than a hundred, and only such numbers will be considered in the remainder of the paper.

Once we have determined the functions $f(v)$ and $g(v)$, we can also run simulations of the corresponding ABP model and measure different kinds of statistics and compare them to the corresponding ones in the CMM model. This will further help us to illustrate the dependence of the approximation's quality on the number of motors $N$. The ABP simulations are done for two sets of functions $f(v)$ and $g(v)$, respectively, given by the increment method (with $N=400$ ) and the distribution method (with $N_{1}=400$ and $N_{2}=300$ ). For the integration of Eq. (1), we use a simple stochastic Euler algorithm [20] with $\Delta t^{\mathrm{sim}}=10^{-4}$.

First, we compare the stationary distribution of the simulated ABP (from just the increment method for the sake of clarity) and the CMM models. As shown in Fig. 5(a), the probability densities are rather close. Small deviations are observed for $N=100$, in particular, around $v=0$ where the probability density of the CMM velocity has a significantly larger maximum than that of the ABP model. However, this discrepancy is less apparent when the ABP is simulated using the functions from the distribution methods. Those differences are an indication that the CMM model cannot be well described by an ABP at small $N$.

To gauge the similarity of the probability distributions, we use the distance between the square roots of the distributions

$$
\gamma_{N}=\frac{1}{2} \int_{v_{\min }}^{v_{\max }} d v\left(\sqrt{P_{0, N}(v)}-\sqrt{\tilde{P}_{0, N}(v)}\right)^{2},
$$

with $v_{\max }=-v_{\min }=60$, and here $\tilde{P}_{0, N}(v)$ is the stationary distribution obtained by the Langevin simulations. This 

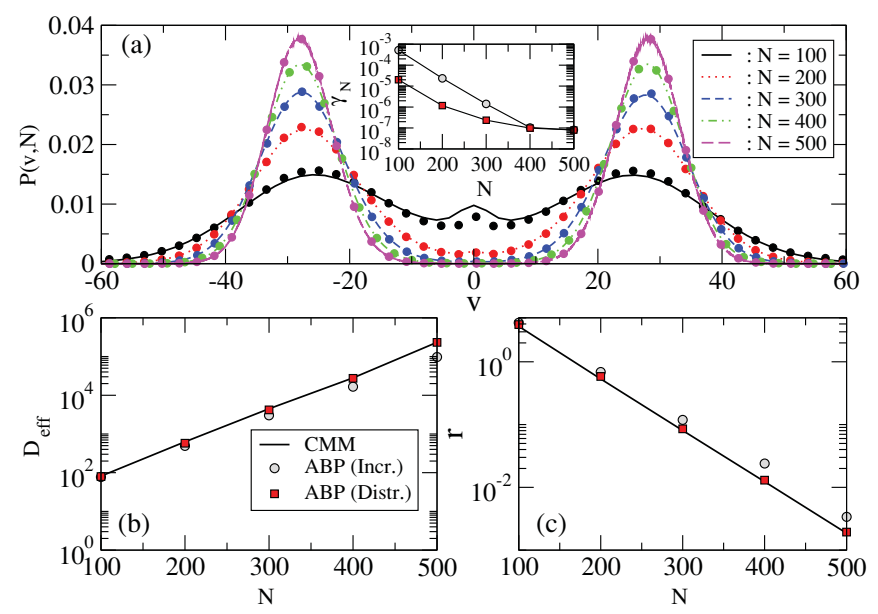

FIG. 5. (Color online) Statistics of the CMM and ABP model for different numbers of motors. (a) Comparison of the normalized distribution for various $N$ in the symmetric case, without external forces, plotted for $N=100,200,300,400$, and 500. Simulation results for the velocity histograms of CMM (lines) and ABP (dots) models; for the latter, we used the increment method for $f(v)$ and $g(v)$. Inset shows the distance between the distributions given by Eq. (27) as a function of $N$. (b) and (c) $D_{\text {eff }}$ and $r$ for various $N$ in a log-normal plot for the two-state (lines) and the ABP (points). Here, the dots and the squares stand for simulation of the ABP with functions extracted from the increments and the distribution, respectively.

function is shown in the inset of Fig. 5(a) for the two sets of functions and reveals an exponential decay of the difference between the distributions. For a large number of motors, the two measures yield similar results, reaching our numerical precision (the latter is set by the expected difference between two independent draws of finite size from the same probability density).

To study the mapping of the CMM to an ABP, we also need to check their respective dynamics. In fact, systems with completely different dynamics can share the same stationary properties. For instance, in our problem a simple shift in the value of $C_{Q}$ will yield different functions $f(v)$ and $g(v)$ but the same distribution; only the diffusion constant will be modified. For this reason, we show in Figs. 5(b) and 5(c) the effective diffusion coefficient [measured via Eq. (17)] and the switching rate between the two metastable velocities which are related to the dynamic properties of the models.

Let us first focus on the comparison between the CMM model (solid lines) and the ABP model with $f(v)$ and $g(v)$ obtained via the distribution method (squares). For the latter we used the value of the diffusion coefficient of the CMM model for $N_{D}=N_{1}=400$ and that is why we can expect to recover the value for $N=400$. However, we also find an excellent agreement between the CMM and the ABP models for both $D_{\text {eff }}$ and $r$ for all values of $N$.

If we use in the ABP model drift term and noise amplitude obtained via the increment method [circles in Figs. 5(b) and 5(c)], the agreement between CMM and the ABP model is less impressive and the relative deviations even grow with $N$. This can be understood as follows. The increment method yields a slightly smaller barrier in the velocity potential [see

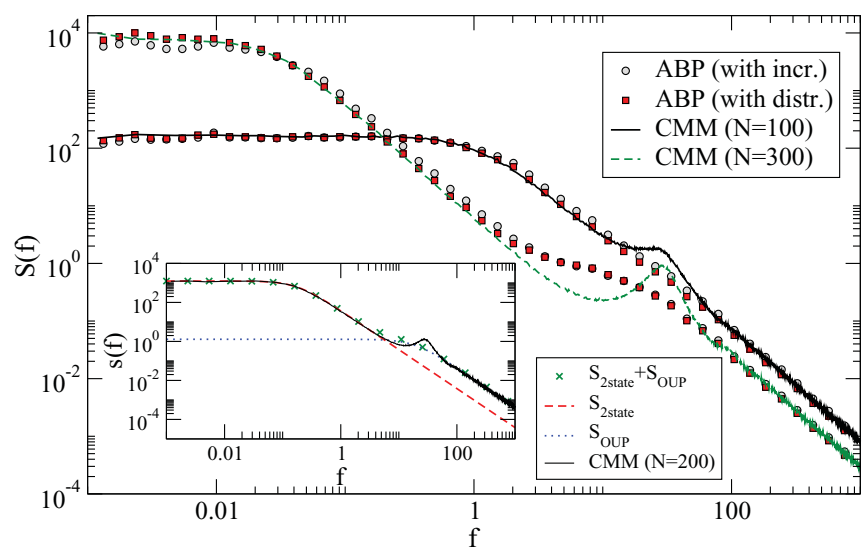

FIG. 6. (Color online) Comparison of the power spectra for $N=$ 100 and 300 of the CMM and the ABP simulated with the results of both methods. In the insert is shown the power spectrum of the CMM with $N=200$ compared to the two Lorentzian approximations given by Eqs. (29) and (31) with the respected measured value for $D_{\text {eff }}$ and $r$.

Fig. 3(a)], which results in higher switching rates. From simple Kramers rate arguments we can expect that the relative difference between two rates for differing barriers increases for decreasing noise intensities, i.e., in our system with increasing $N$. We may conclude that with respect to diffusion coefficient and switching rate, the distribution method provides the more faithful ABP description of the CMM model.

Finally, to fully understand the relevance of this mapping, we may also ask whether the ABP and CMM models agree with respect to their correlation statistics. In particular, we look at the power spectrum of the velocity (the Fourier transform of the autocorrelation function of the velocity), defined by

$$
S(f)=\lim _{T \rightarrow \infty} \frac{\left\langle\tilde{v} \tilde{v}^{*}\right\rangle}{T}, \quad \tilde{v}=\int_{0}^{T} d t v(t) e^{2 \pi i f t},
$$

where the asterisk denotes the complex conjugate. The power spectrum, as determined from $\mathrm{ABP}$ and $\mathrm{CMM}$ simulations, is shown in double logarithmic scaling in Fig. 6. For all numbers of motors and for both models, spectra reveal a Lorentzian shape at low frequencies $(f<1)$. However, some discrepancies arise at very low frequency between the two ABP simulations. The power spectrum is systematically underestimated with the increment method, whereas the distribution method gives a very good agreement with the CMM power spectrum. This is expected and consistent with the results for the effective diffusion constant, because according to the Green-Kubo relation, $S(0)=2 D_{\text {eff }}$. To describe the long time behavior of the CMM by means of an ABP, it is then clear that the distribution method is better suited. If we assume a Markovian two-state process for the velocity jumping between two discrete values $v_{-}$and $v_{+}$with rate $r$, the spectrum obeys

$$
S_{2 \text { state }}(f)=\frac{2 D_{\text {eff }}}{1+(\pi f / r)^{2}},
$$

where we have used that the spatial diffusion coefficient for such a process would be $D_{\text {eff }}=v_{ \pm}^{2} /(2 r)$. Using the switching rate and diffusion coefficient measured in the CMM and ABP simulations (see Fig. 5), this formula yields an 
excellent agreement with the numerically determined spectra (dashed line in the insert of Fig. 6) for low to moderate frequencies.

The behavior at moderate to very high frequencies is more complicated and also reveals a basic difference between the CMM and ABP models. Let us first point out that deviation from the spectrum of a two-state process can be expected for both models at very high frequencies. This is because the velocity in both models is a continuous (ABP) or multi-state (CMM) variable, the fluctuations of which at very short time scales (high frequency) are dominated by fluctuations around the stable fixed points. Although these fluctuations are naturally correlated to the switching process, which is described by the two-state approximation, the two contributions to the power spectrum may be approximated by two Lorentzians,

$$
S \approx S_{2 \text { state }}+S_{\text {OUP }}
$$

one for the two-state process, and one for an approximate Ornstein-Uhlenbeck process performed around the potential minima,

$$
S_{\text {OUP }}=\frac{2 g^{2}\left(v_{\min }\right) / N}{f^{\prime}\left(v_{\min }\right)^{2}+(2 \pi f)^{2}},
$$

where we have approximated the noise by an additive one with a noise intensity $g\left(v_{\min }\right)^{2} / N$. Using the noise intensity and the curvature of the potential at the potential minimum $v_{\text {min }}$, we observe a reasonable agreement between the spectra of the CMM simulations and Eq. (31) at high frequencies (dotted line in the insert of Fig. 6). If we now look at the total spectrum given by Eq. (30), the CMM model yields a very good agreement to both high and low frequencies (stars in the inset of Fig. 6) but shows an additional feature at moderately high frequencies: there is a peak at around $f \approx 25$ which is not predicted by our simple approximation Eq. (30) and not displayed by the ABP model. For large $N$, the peak becomes sharper and can be regarded as a small-amplitude stochastic oscillation which is present in the dynamics of the CMM model. We discuss this qualitative difference between the ABP and CMM models below in Sec. IV D.

In conclusion, we have seen that the probability density, the rate of velocity reversals, the spatial diffusion coefficient, and most features of the power spectrum are in excellent agreement for CMM and ABP models. This confirms that within the limit of large $N$, the ABP model becomes a good approximation of the CMM model in the stationary state. However, there is one specific feature that becomes manifest in the power spectrum which is not captured by the ABP approximation: the CMM model generates high-frequency oscillations in the potential minima which lead to a peak in the power spectrum.

\section{B. Asymmetric cases $\left(a \neq L / 2\right.$ or $\left.F_{\text {ext }} \neq 0\right)$}

In general, the system of coupled molecular motors will not be spatially symmetric, and so it is worth asking how the functions $f(v), g(v)$ will change if we apply either a bias force $F_{\text {ext }}>0$ or break the intrinsic symmetry by breaking the reflection symmetry of the spatial potential $W(x)$, i.e., choose $a \neq L / 2$.

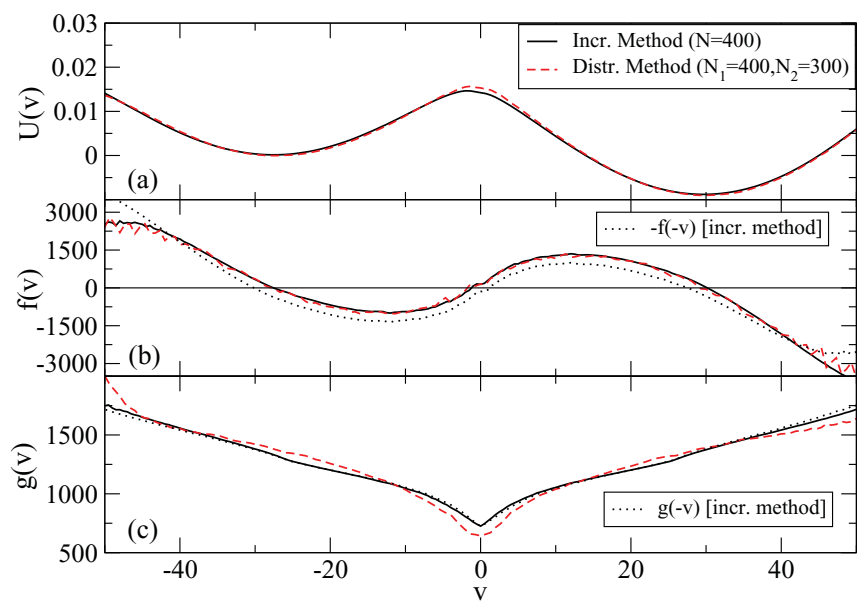

FIG. 7. (Color online) Effective potential (a), drift (b), and noise amplitude (c) of the CMM model with an additional bias $F=0.02$ [symmetric potential $W(x)$ with $a=L / 2$ ]. Functions were determined by the distribution method (with $N_{1}=400, N_{2}=300$, dashed line) and increment method (with $N=400$, solid line). Dotted lines are the mirrored functions $-f(-v)$ and $g(-v)$, shown to highlight a potential asymmetry of the curves.

Effective velocity potential and the functions $f(v), g(v)$ are shown in Fig. 7 for a finite value of the external force $F_{\text {ext }}=0.02$. The potential becomes asymmetric in this case; in particular, the potential well corresponding to positive velocities becomes deeper and thus more stable. There are slight shifts in the locations of potential minima $\left(v_{-} \approx\right.$ $\left.-28, v_{+} \approx 30\right)$ and maximum $\left(v_{\max } \approx-1\right)$.

The main effect of the external bias on the function $f(v)$ is an upward shift. This is revealed by comparing the function to its mirrored version $-f(-v)$ [dotted line in Fig. 7(b)]; in a spatially symmetric system these two functions should agree. For a positive bias, however, the function $-f(-v)$ is lower than $f(v)$, and for the larger part of the velocity range shown, the difference between the two lines (solid and dotted ones) can be regarded as a velocity-independent shift. Only at extreme values of the velocity, the difference seems to vanish. On the other hand, there is no appreciable effect of the force on the noise amplitude $g(v)$, except for extreme values of the velocity.

In Fig. 8 we show the effective velocity potential and the functions $f(v)$ and $g(v)$ for an asymmetric potential without external force (here we choose $a=0.48 L$ ). Just as in the previous case, the symmetry breaking in the problem is reflected in the effective potential $U$, and the well corresponding to negative velocities becomes deeper and thus more stable. The main effect of the asymmetry is again to shift the locations of the minima $\left(V_{-} \approx=-30, v_{+} \approx 28\right.$ ) and maximum $\left(v_{\max } \approx 1\right)$. We have chosen the asymmetry to mirror the effect obtained with the external force.

The effect of the asymmetry over the functions $f(v)$ and $g(v)$ is of the same type as before. The function $f(v)$ as a whole is shifted down and $g(v)$ is left unchanged. Over a relatively large range of velocities, the effect of the asymmetry can be regarded as a velocity-independent shift.

The presence of an asymmetric potential seems to have the same type of effect as applying an external force directly on the backbone. This result is consistent with [17], where the 


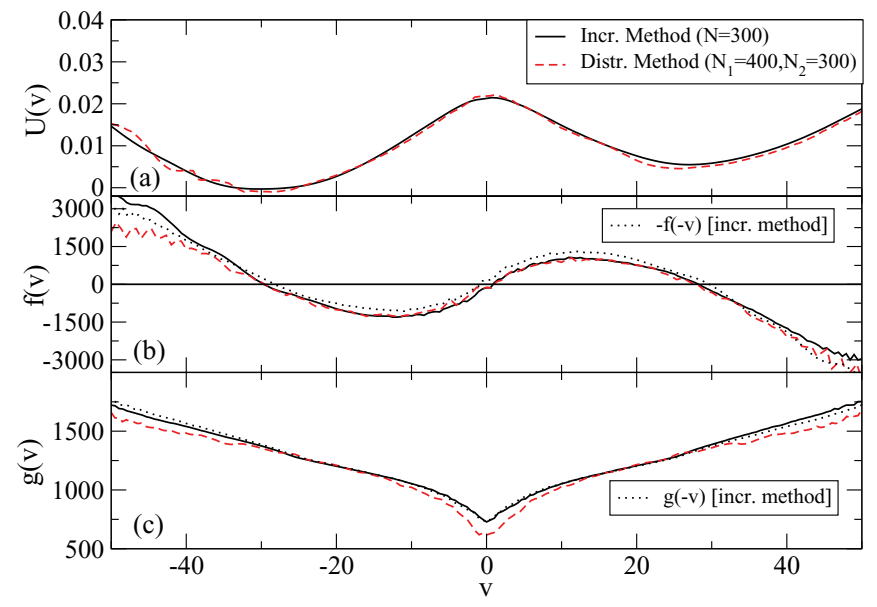

FIG. 8. (Color online) Effective potential (a), drift (b), and noise amplitude (c) of the CMM model with an asymmetric spatial potential $a=0.2 L$ (no external force, $F_{\mathrm{ext}}=0$ ), determined by the distribution method (with $N_{1}=300, N_{2}=400$, dashed line) and increment method (with $N=400$, solid line).

authors recovered the bidirectionality of the motion as well as the giant diffusion properties of the symmetrical case by applying a force to an asymmetric potential. A detailed study of this effect would be interesting. However, the simple case of an external force in a symmetric potential is already very rich, and we now focus on some aspects of this.

\section{Drift function $f(v)$ and the nonlinear force-velocity relation}

For the CMM model Jülicher et al. calculated a nonlinear force-velocity relation analytically in the thermodynamic limit $N \rightarrow \infty$. This relation Eq. (8) gives us for any value of the backbone's velocity the required external force. In terms of our general drift function $f\left(v ; F_{\text {ext }}\right)$, this relation corresponds to the solution

$$
f\left(v ; F_{\text {ext }}\right)=0 .
$$

Because the relation $F_{\text {ext }}=\hat{f}(v)$ has the symmetric shape of an inverted $\mathrm{N}$, one may wonder whether it is proportional to the nonlinear drift $f(v)$ at $F_{\text {ext }}=0$, i.e., to $f_{0}(v)=f\left(v ; F_{\text {ext }}=0\right)$.

In general, it seems quite hopeless to infer the function $f_{0}(v)$ from the force-dependent zeros of the function $f\left(v ; F_{\text {ext }}\right)$. However, both functions would be proportional if the external force would enter in an additive way in the drift function

$$
f\left(v ; F_{\text {ext }}\right)=f_{0}(v)+\alpha F_{\text {ext }},
$$

where we abbreviated $f_{0}(v)=f\left(v, F_{\mathrm{ext}}=0\right)$. In this case, clearly

$$
f_{0}(v)=-\alpha F_{\mathrm{ext}}=\alpha \hat{f}(v),
$$

where the latter function is analytically given in Eq. (8). A bias force of the motor system would thus imply only a bias force in the approximate ABP dynamics. This is plausible when we look at the vertical shift of the curve $f(v)$ (Fig. 7) introduced
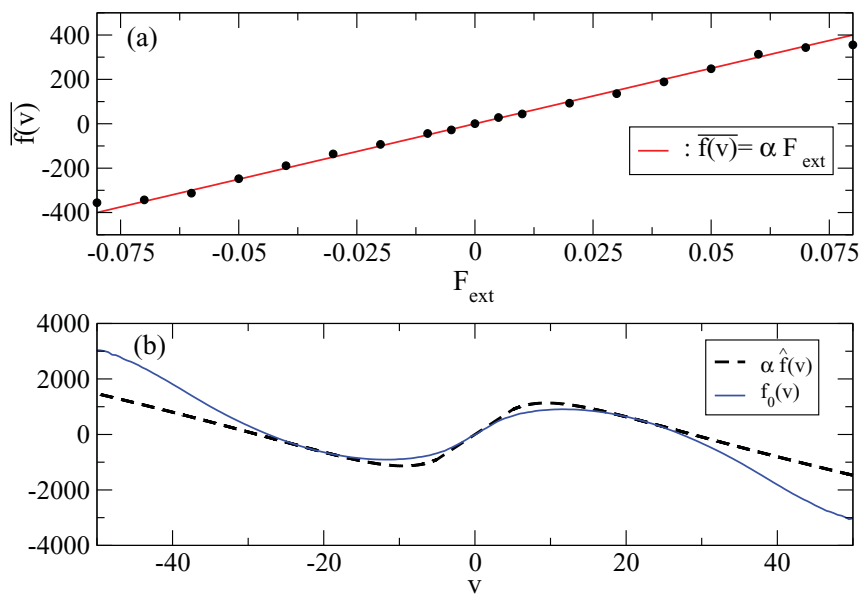

FIG. 9. (Color online) (a) Mean shift of the drift function vs external force for $N=500$ motors. The solid line gives a linear fit with $\alpha \approx 4937$. (b) Comparison of the nonlinear drift and the scaled force-velocity relation [Eq. (8) in Sec. II].

by a bias force. We can extract the slope $\alpha$ by estimating the mean shift as follows:

$$
\overline{f\left(v, F_{\mathrm{ext}}\right)}=\frac{1}{v_{\max }-v_{\min }} \int_{v_{\min }}^{v_{\max }} d v f\left(v, F_{\mathrm{ext}}\right) .
$$

If we plot this average as a function of the external force [see Fig. 9(a)], we indeed observe a linear relationship as long as the forces are not too strong, i.e., as long as we still observe velocity reversals. The slope $\alpha \approx 5000$ can now be used to compare $\alpha \hat{f}(v)$ to $f_{0}(v)$. This slope can be also interpreted as the average of the derivative $\partial f\left(v, F_{\text {ext }}\right) /\left.\partial F_{\text {ext }}\right|_{F_{\text {ext }}=0}$, with respect to velocity.

The two functions [cf. Fig. 9(b)] share some similarity: both are shaped like an inverted $\mathrm{N}$, and their magnitude and their zeros agree. One can thus regard $\alpha\left[\hat{f}(v)+F_{\text {ext }}\right]$ as an approximation of $f\left(v, F_{\text {ext }}\right)$. We also note, however, that several of these features can be expected. For instance, we made the magnitude of both functions similar by the factor $\alpha$ that so far cannot be calculated analytically and has to be determined in simulations. Furthermore, the fact that the zeros of both functions are the same is likewise built in because they yield the stable and unstable solutions for the velocity, which are correctly predicted by the thermodynamic limit. We can also state that there is quantitative disagreement, in particular, at higher velocities, where the functions differ by almost a factor of two.

Although the static force-velocity relation from the thermodynamic limit gives a rough approximation of the nonlinear drift, more analytical efforts are required to calculate $f\left(v, F_{\text {ext }}\right)$ accurately.

\section{Limitation of the ABP model - relaxation from an extreme value of the velocity and high-frequency behavior}

In order to understand the presence of a peak in the power spectrum at $f \approx 25$ for the CMM model (see Fig. 6), we now inspect the temporal evolution of the probability density of motors. 
It is not hard to calculate the infinitesimal moments of the velocity at a given time if we know the probability density of the motors. Of course, knowing this density is really the main difficulty of the entire problem; however, there are special cases for which we know it. It is easy to see that the maximal possible velocity in the system of motors is achieved when all the motors which are on the left slope are in state 1, while those located on the opposing slope are in state zero, i.e.,

$$
\begin{aligned}
& P_{1, v_{\max }}(x)=\Theta(a-x) / L, \\
& P_{0, v_{\max }}(x)=1 / L-P_{1}(x) .
\end{aligned}
$$

In this case, the potential will exert the maximum possible positive force on the backbone and we know then the maximum possible velocity, which will be

$$
v_{\max }=\frac{1}{\lambda}\left[F_{\text {ext }}+\frac{\hat{W}}{L}\right]
$$

(the asymmetry parameter $a$ does not enter this relation).

Let us assume that we take a very large number of motors and prepare the system in this state of maximal positive velocity. If Eq. (1) would also apply in this situation, then $v(t)$ should relax from the maximal value to the metastable velocity, corresponding to the first zero $v_{+}$of $f(v)$ on the right $\left[f\left(v_{+}\right)=0\right.$ with $\left.v_{+}>0\right]$. For a finite number of motors, we expect to find some small fluctuations around this relaxation curve.

What we find instead of a monotonic relaxation is a damped oscillation. As shown in Fig. 10(a) for $N=500$, the motor assembly quickly reaches a state of a very small but positive velocity, then goes back to larger positive velocities and oscillates around the expected steady-state value $v_{+}$, which is finally approached (there remain, of course, finite-size

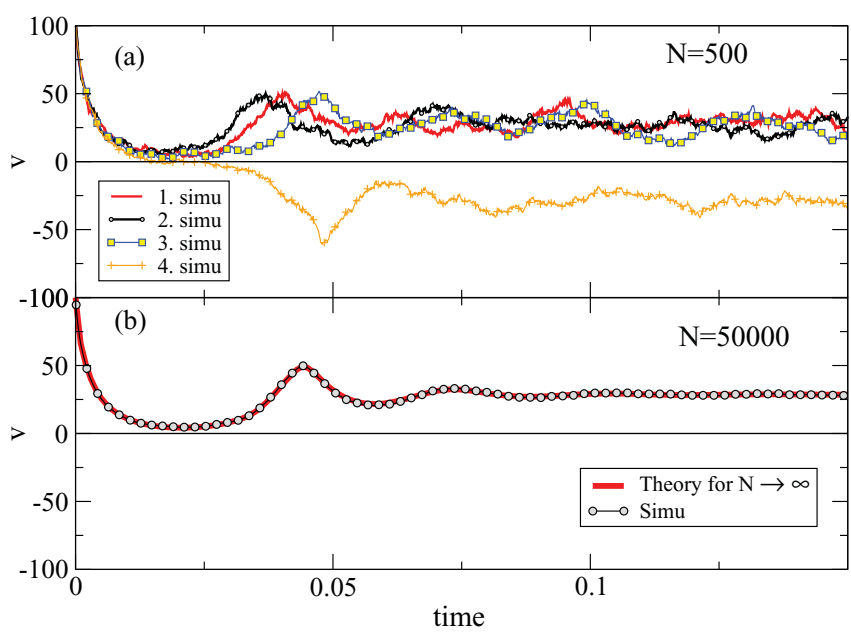

FIG. 10. (Color online) Relaxation of the velocity from an extreme value. (a) Several simulations for $N=500$ show a damped stochastic oscillation with a considerable variability among the realizations; one of the simulations makes a transition to negative velocities. (b) For a much higher number of motors $(N=50000)$, the variability of the transient is strongly reduced (not shown); the one simulation we show (dashed line) agrees well with what we obtain as the transient velocity from the time-dependent numerical solution of the thermodynamic-limit equation, Eq. (4) (solid line). fluctuations around $v_{+}$). When the velocity is close to zero, its time course is apparently rather sensitive to noise. For that reason, the timing of the first peak (around $t \approx 0.04$ ) varies considerably among the different realizations. Moreover, one realization (marked 4. simulation) even makes a transition to negative velocities; we have verified by more realizations that for $N=500$ this occurs about one out of six times.

By increasing the number of motors to $N=50000$ [Fig. 10(b)], fluctuations are strongly reduced. The resulting curve is also compared to the velocity computed from the time-dependent solution of Eq. (4) with the initial condition Eq. (36); the agreement is excellent. This verifies that the damped oscillatory relaxation is not a feature that is introduced by the finite number of motors.

This damped oscillatory process appears with a period of $T \approx 0.04$, which corresponds exactly to the peak frequency measured in the power spectrum at $f \approx 25$. Moreover, by increasing the number of motors, the oscillations become more stable and less noisy, which is in complete agreement with our observation of the power spectrum (the peak becomes more apparent with an increased number of motors). This indicates that the relaxation observed here for the case of an extreme velocity always affects the dynamics and thus is responsible for the peak in the power spectrum.

\section{SUMMARY AND CONCLUSION}

Our study has confirmed that for the molecular motor system proposed in [16] and studied numerically for a finite number of motors in [15] and [17], an approximation by an active Brownian particle model is meaningful. We have determined the specific drift function and noise amplitudes via two independent methods which yielded a close agreement. The mapping from the CMM model to the ABP model works in the symmetric case but also with an additional force or an intrinsic asymmetry of the motor model.

The nonlinear drift we found in this way revealed an inverted N shape. Qualitatively, this could be expected from the force velocity relation that was previously found in the thermodynamic limit of the model [16]. In this paper, we showed, however, that the latter does not completely agree in detail with the drift function $f(v)$. In order to find the function $f(v)$ analytically, we have to employ methods beyond the steady-state solution for finite force $F_{\text {ext }}$ found in the thermodynamic limit by Jülicher and Prost.

Surprisingly, the noise amplitude we found by the two methods depends on the velocity. Noise increases for higher speed. This is in contrast to the system of Brownian particles globally coupled via a velocity alignment, which was studied in [14]. There the noise amplitude is largest for vanishing velocity and decreases with increasing speed monotonically. We can conclude that the speed dependence of the noise amplitude relies on the particularities of the system under study.

We also found a clear limitation of the ABP approximation for the motor model; indeed, it fails to reproduce a peak that appears in the power spectrum of the CMM's dynamics. We illustrated this by looking at the extreme case of the system, starting with the maximal possible velocity (which uniquely determines the biochemical state of each motor). There we 
did not find the monotonic relaxation into the steady state but a damped oscillation of the velocity which occurs at the frequency of the peak observed in the power spectrum. In order to describe this process, at least one additional degree of freedom is required for any given value of $N$.

This study was limited to the case of vanishing thermal fluctuations in the CMM model [ $T=0$ in Eq. (2)]. Preliminary simulation results of the symmetric case indicate that for a large number of motors additional thermal fluctuations do not change significantly the functions $f(v)$ and $g(v)$ obtained via a modified increment method. However, it is also possible that in other parameter regimes sufficiently strong thermal fluctuations may facilitate the velocity reversals or, in the extreme case, even destroy the bidirectionality of the motion completely.

Our study underpins the importance of the ABP model in describing biological motility. The open question is how the functions $f(v)$ and $g(v)$ can be derived analytically from the CMM model. We regard our efforts as an encouragement for researchers to work out analytical methods for this interesting problem.

\section{ACKNOWLEDGMENT}

We thank Frank Jülicher for valuable discussions on the topic of this paper.
[1] J. Howard, Mechanics of Motor Proteins and the Cytoskeleton (Sinauer Associates, Sunderland, MA, 2001).

[2] D. Bray, Cell Movements (Garland, New York, 2001).

[3] H. Chaté, F. Ginelli, G. Gregoire, F. Peruani, and F. Raynaud, Eur. Phys. J. B 64, 451 (2008).

[4] S. Ramaswamy, Annu. Rev. Conden. Ma. P. 1, 323 (2010).

[5] T. Vicsek, A. Czirok, E. Ben-Jacob, I. Cohen, and O. Shochet, Phys. Rev. Lett. 75, 1226 (1995).

[6] M. Schienbein and H. Gruler, Bull. Math. Biol. 55, 585 (1993).

[7] F. Schweitzer, W. Ebeling, and B. Tilch, Phys. Rev. Lett. 80, 5044 (1998).

[8] U. Erdmann, W. Ebeling, L. Schimansky-Geier, and F. Schweitzer, Eur. Phys. J. B 15, 105 (2000).

[9] F. Schweitzer, Brownian Agents and Active Particles (Springer, New York, 2002).

[10] Yu. L. Klimontovich, Statistical Theory of Open Systems (Kluwer Academic Publishers, Dordrecht-Boston-London, 1995).
[11] B. Lindner, New. J. Phys. 9, 136 (2007).

[12] D. Selmeczi, S. Mosler, P. H. Hagedorn, N. B. Larsen, and H. Flyvbjerg, Biophys. J. 89, 912 (2005).

[13] D. Selmeczi, L. Li, L. I. I. Pedersen, S. F. Norrelykke, P. H. Hagedorn, S. Mosler, N. B. Larsen, E. C. Cox, and H. Flyvbjerg, Eur. Phys. J-Spec. Top. 157, 1 (2008).

[14] C. A. Yates, R. Erban, C. Escudero, I. D. Couzin, J. Buhl, I. G. Kevrekidis, P. K. Maini, and D. J. T. Sumpter, Proc. Natl. Acad. Sci. USA 106, 5464 (2009).

[15] M. Badoual, F. Jülicher, and J. Prost, Proc. Natl. Acad. Sci. USA 99, 6696 (2002).

[16] F. Jülicher and J. Prost, Phys. Rev. Lett. 75, 2618 (1995).

[17] B. Lindner and E. M. Nicola, Phys. Rev. Lett. 101, 190603 (2008).

[18] S. Endow and H. Higuchi, Nature 406, 913 (2000).

[19] P. Reimann, Phys. Rep. 361, 57 (2002).

[20] H. Risken, The Fokker-Planck Equation (Springer, Berlin, 1989).

[21] D. T. Gillespie, J. Chem. Phys. 113, 297 (2000). 